\title{
Mehr Kohärenz für eine wirksame Suchtpolitik
}

\author{
Irene Abderhalden, Monique Portner-Helfer, Frank Zobel
}

Sucht Schweiz

Die Suchtpolitik ist durchzogen von Widersprüchen und Inkohärenzen. Der Alkoholkonsum im öffentlichen Raum stört, während der Alkoholmarkt weiter liberalisiert wird. Die Politik will das Glücksspielangebot im Internet erweitern, und gleichzeitig überlegt man, die Mittel für die Prävention der Glücksspielsucht zu streichen. Die Zuwendungen an die Tabakproduktion bleiben gleich hoch wie die Mittel für die Tabakprävention. Zudem treffen konsequentere Einschränkungen der Werbung, so wie sie anderswo in Europa üblich sind, hierzulande immer noch auf grossen Widerstand.

Diese Widersprüche schmälern die Glaubwürdigkeit der Suchtpolitik in der Schweiz. Diese scheint eher auf ideologischen Haltungen aufzubauen und nicht auf den Folgen, die sie für betroffene Personen, ihr Umfeld und die Gesellschaft hat. Die Gelegenheit wäre nun da, um einen glaubwürdigen und effizienten Ansatz für den Suchtbereich zu entwickeln.

Zum ersten Mal prüfen die beiden Parlamentskammern die Gesetzgebungen zu Alkohol, Tabakerzeugnissen und Glücksspiel parallel. Zudem haben sich die Parlamentarierinnen und Parlamentarier auch noch mit Anfragen von Städten und Kantonen zur Cannabisfrage zu befassen. Die drei Gesetze werden jedoch behandelt, als hätten sie nichts miteinander zu tun. Und um die Inhalte jedes dieser Gesetze feilschen Interessengruppen. Präventionsanliegen haben einen schweren Stand: Gelder für Prävention und Behandlung drohen gestrichen zu werden, während die

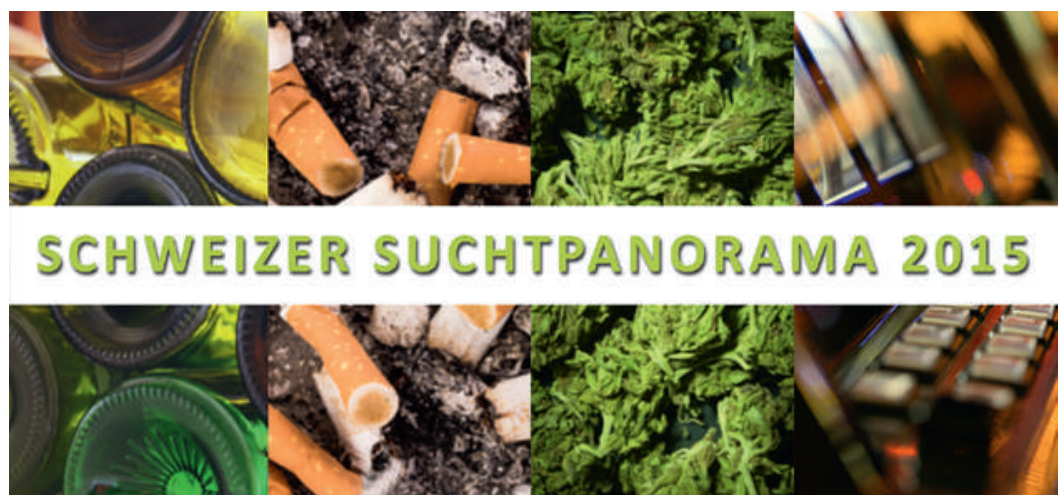

Thematisiert die Vielfalt der Süchte: das Logo des Schweizer Suchtpanoramas. erwiesenermassen wirksamen strukturellen Massnahmen, welche die Erhältlichkeit und Attraktivität eines Suchtmittels einschränken, kein Gehör finden. Suchtfachpersonen weisen zu Recht immer wieder darauf hin, dass Suchtprobleme nicht nur Probleme

Zum ersten Mal prüfen beide Parlamentskammern Gesetzgebungen zu Alkohol, Tabakerzeugnissen und Glücksspiel parallel.

einzelner Individuen sind, sondern die gesamte Gesellschaft betreffen. Oft blendet die öffentliche Diskussion aus, dass der Markt das Verhalten von Individuen, gerade von besonders gefährdeten Personen, stark beeinflusst. Der Erhältlichkeit und der Anpreisung von Alkohol, Tabak oder Glücksspielen Grenzen $\mathrm{zu}$ setzen würde eigentlich nur bedeuten, einen Markt zu regulieren, den man in letzter Zeit mehr und mehr sich selbst überlassen hat und dadurch dringend einer Korrektur bedarf. Will die Gesellschaft Suchtprobleme glaubwürdig und nachhaltig reduzieren, wird die Politik den Markt wirksamer regulieren und parallel dazu der Prävention, der Schadensminderung und der Behandlung Mittel zur Verfügung stellen müssen.

\section{Suchtbereich zu Beginn des 21. Jahr- hunderts im Umbruch}

Die Entwicklungen bei den verschiedenen Substanzen sowie beim Glücksspiel weisen je spezifische Eigenheiten auf. Geänderte Normen beim Alkohol- und Tabakkonsum, neue Angebote im Bereich Glücksspiel und illegale Drogen sowie Schritte zur Markterweiterung prägen das Geschehen.

\section{Weniger Alkohol, aber immer noch viele Alkoholräusche}

Im vergangenen Jahrzehnt ist in der Schweiz wie auch in anderen Ländern der Alkoholkonsum gesunken. Bestimmte Formen des täglichen Trinkens sind nach und nach verschwunden und finden sich heute vor allem noch bei älteren Personen. Eine Folge davon ist eine Abnahme des Gesamtkonsums in der Schweiz, insbeson- 
dere von Wein. Die Abnahme des täglichen Alkoholkonsums bei den Männern hat zu einer Annäherung zwischen den Geschlechtern beigetragen. Gleichzeitig trinken heute mehr Frauen regelmässig Alkohol als noch vor 20 Jahren, vor allem die 65- bis 74-jährigen.

Der Gesamtkonsum von Alkohol und der Risikokonsum haben bei Jugendlichen und jungen Erwachsenen zwischen 15 und 24 Jahren in den vergangenen Jahrzehnten zugenommen. Die punktuellen Rauschzustände stagnieren heute auf einem hohen Niveau. Ohne Zweifel haben die Ausdehnung der Verkaufszeiten und der nächtlichen Freizeitangebote sowie die Preissenkung von Alkoholika hierzu beigetragen. Exzessiver Alkoholkonsum findet sich indes in allen Alterskategorien. Er ist auch bei berufstätigen Männern und solchen mit hohem Einkommen stärker verbreitet.

\section{Präventionsanliegen haben einen schweren Stand: Gelder für Prävention und Behandlung drohen gestrichen zu werden.}

\section{Der Tabak: das Ende der Baisse?}

Der Gebrauch von Tabak ist unter dem Einfluss struktureller Massnahmen und sozialer Normänderungen gesunken. Das Viertel der Bevölkerung, das noch raucht, tut dies heute oft nur noch auf der Strasse und auf Balkonen. Studienergebnisse deuten aber darauf hin, dass die Abwärtsentwicklung bei der Anzahl Raucher und Raucherinnen und beim Passivrauchen nun zu Ende sein könnte.

\section{Illegale Drogen: weniger Heroin, mehr Ecstasy} Der dritte wichtige Abwärtstrend betrifft ein Phänomen, das lange im Zentrum der öffentlichen Aufmerksamkeit stand: der Konsum von Heroin. Es ist unter anderem der besseren Behandlung und Betreuung der Konsumierenden zu verdanken, dass sich die Problematik in Zusammenhang mit illegalen Drogen in der Schweiz entschärft hat. Allerdings konsumieren mindestens 40000 jüngere Menschen täglich Cannabis, manche von ihnen von morgens bis abends. Der illegale Markt ist zudem in dauernder Veränderung, wie sich z.B. mit der Rückkehr von Ecstasy zeigt. Ausserdem entwickelt sich der Verkauf übers Internet, insbesondere von neuen psychoaktiven Substanzen.

\section{Viele Glücksspiele, viele Verlierer}

Das Angebot an Glücksspielen in der Schweiz ist zu Beginn dieses Jahrhunderts stark gestiegen, einerseits wegen der Zulassung von Casinos und andererseits durch die Diversifizierung der Lotteriespiele. Heute hat die Schweiz eine der grössten Casinodichten der Welt. Die Anzahl exzessiv Spielender und die mit dem Glücksspiel verbundenen sozialen Kosten sind sehr hoch, und die Forschung konnte bereits nachweisen, dass bei Glücksspielen im Internet noch höhere Risiken bestehen als bei herkömmlichen Glücksspielen.

\section{Einige Konsumstile ändern sich, aber die Mehrzahl der Folgeprobleme bleibt}

Heute kann man rund um die Uhr Alkohol kaufen, oft zu niedrigen Preisen, oder im Internet um Geld spielen. Auch zu Cannabis, Kokain oder Ecstasy kommt man in Schweizer Städten schnell und relativ problem los.

Trotz erfreulichen Konsumentwicklungen in manchen Bereichen bleibt die Problemlast hoch. Der Tabak ist die wichtigste Ursache für frühzeitige Todesfälle in der Schweiz; der Alkohol folgt an dritter Stelle. Psychoaktive Substanzen und das Glücksspiel hängen jedes Jahr mit insgesamt mehr als 10 ooo Todesfällen zusammen und mit sozialen Kosten, die 10 Milliarden Franken übersteigen. Erwähnt werden müssen auch das Leid der mehreren Hunderttausend abhängigen Menschen sowie der Nahestehenden und die Folgeprobleme, welche mehr als die Hälfte der Bevölkerung betreffen. Die Suchtpolitik braucht daher neue Impulse.

\section{Forderungen nach strukturellen Massnahmen}

Die laufende Revision des Alkoholgesetzes bietet die Chance, den problematischen Konsum zu vermindern. Griffige und erwiesenermassen wirksame Massnahmen wie höhere Preise und kürzere Verkaufszeiten (Nachtverkaufsverbot) sind im Sinne von Marktkorrekturen notwendig bzw. "not-wendend». Beim neuen Tabakproduktegesetz steht für Sucht Schweiz ein nationales, streng kontrolliertes Abgabeverbot an Minderjährige sowie ein umfassendes Werbe- und Sponsoringverbot im Fokus. Zudem sollten sowohl Zigarettenautomaten als auch die Verkaufsförderung durch Rabatte unzulässig sein. Bei der Debatte rund um das neue Geldspielgesetz setzt sich Sucht Schweiz für einen besseren Spielerschutz ein, einschliesslich eines Verbots von Gratisspielen und einer griffigeren Werberegulierung. Auch müssen den Kantonen die Gelder zur Prävention und Behandlung der Spielsucht zugesprochen werden, damit sie den entsprechenden gesetzlichen Auftrag auch erfüllen können. Beim Cannabis begrüsst Sucht Schweiz das Prüfen neuer Regulierungsmodelle wie beispielsweise das am weitesten fortgeschrittene Projekt in Genf, das den legalen Cannabiszugang für Erwachsene im Rahmen von Konsumvereinigungen evaluieren will. 